\title{
Caractérisation de l'habitat naturel d'une espèce de géophyte menacée (Tulipa sylvestris L.) dans la fôret de Bouhriz (ouest Algérien)
}

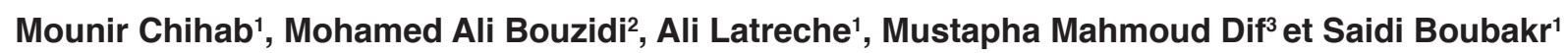

'Laboratoire de Biodiversité Végétale Conservation et Valorisation, faculté des sciences de la nature et de la vie, université Djilali Liabes de Sidi bel Abbes, 22000. Algérie.

'Laboratoire d'écodéveloppement des espaces, Faculté des sciences de la nature et de la vie, université Djilali Liabes de Sidi Bel Abbes, 22000Algérie.

${ }^{3}$ Département des sciences de la nature et de la vie, centre universitaire Nour Bachir, El Bayad, 32000. Algérie.

\section{Correspondencia}

M. Ali Bouzidi

e-mail: medalibouzidi@yahoo.fr

Recibido: 6 enero 2018

Aceptado: 20 marzo 2018

Publicado on-line: octubre 2018

\begin{abstract}
Résumé
Ce travail consiste à évaluer l'habitat naturel et écologique de Tulipa sylvestris L. espèce menacée et rare, localisée dans la forêt de Bouhriz (région de Sidi Bel Abbes). Pour cela, quatre stations réparties dans la partie Sud, Sud-est et Nord-est de la forêt ont été choisies en fonction de la présence des géophytes, sur lesquels des prélèvements du sol étaient analysés et des relevés floristiques ont été effectués. En effet, Tulipa sylvestris L. est localisée dans la partie Sud (station 3 uniquement), les résultats pédologiques ont montré que l'espèce est liée à une texture limono-sableuse, peut humide et un $\mathrm{pH}$ légèrement alcalin, moyennement riche en matière organique avec un sol modérément calcaire. L'analyse floristique et statistique par l'analyse factorielle des correspondances (AFC) indiquent une importante richesse de l'ordre de 50 taxons soumis à une forte thérophytisation, un indice de perturbation élevé, justifiant ainsi la rareté et la vulnérabilité de Tulipa sylvestris L. dû essentiellement à l'action anthropique et au surpâturage associé aux incendies.
\end{abstract}

Mots-clés: Tulipa sylvestris L., foret Bouhriz, Algérie, anthropisation.

\section{Introduction}

Le Bassin Méditerranéen constitue l'un des «Hotspots» de la biodiversité planétaire (Myers et al., 2000) principalement parce qu'il bénéficie d'une flore très riche, comptant 25000 espèces de plantes vasculaires, soit $10 \%$ de la flore du globe (Greuter, 1991). Cette richesse végétale est due 
à une grande hétérogénéité spatiale des facteurs géomorphologiques, bioclimatiques et historiques et donc due à une grande hétérogénéité spatiale des habitats naturels (Quézel, 1985).

Les forêts méditerranéennes constituent un milieu naturel fragile perturbé par des utilisations multiples. Les agressions qu'elles ont subies ont cependant variés en fréquence et en intensité au cours des âges en fonction de la démographie humaine, ce qui a déterminé des phases de progression ou régression de leurs surfaces (Marc, 1916; Quézel \& Barbéro, 1990), cette région est aussi le siège d'impacts anthropiques considérables et les points chauds de biodiversité font aussi partie des zones les plus menacées par l'anthropisation (Médail \& Diadema, 2006) dans certaines régions du globe comme le bassin méditerranéen où les enjeux de préservation de la biodiversité sont majeurs (Sala et al., 2000). L'étude de la flore méditerranéenne, en général, et celle de l'Oranie en particulier, a été abordée par plusieurs auteurs durant ces dernières décennies telles que (Ben Abdallah, 2010), (Hadjadj Aoul, 1995), (Alcaraz, 1980), (Guinochet, 1980), (Quézel, 1964). Cependant, le manque de connaissances générales sur la biologie des espèces rares, en particulier végétales, a été fréquemment souligné (Kunin \& Gaston, 1993 ; Murray et al., 2002), le but est de mieux comprendre les processus liés à la régression des populations et d'évaluer ainsi le devenir de leurs populations dans le cadre d'une gestion optimale (Verlaque et al., 2001 ; Lavergne et al., 2003 ; 2005).

C'est dans ce concept que s'inscrit notre étude qui consiste à caractériser l'écologie et l'habitat de Tulipa sylvestris L. qui est une espèce menacée et rare au niveau de l'Algérie d'où la protection est intégrale et de déterminer les menaces et les mesures de la préserver.

\section{Matériel et méthode}

\section{Présentation de la zone d'étude}

Couvrant une superficie de $70 \mathrm{Ha}$, la forêt de Bouhriz se situe à $15 \mathrm{~km}$ de chef-lieu de la wilaya de Sidi Bel Abbes (Algérie occidentale). Elle appartient à la commune d'Amarnas et fait partie de la forêt domaniale de Tenira. Elle est limitée par la forêt de Moksi à l'est, la forêt de Tilmouni au nord, au nord-est par la forêt de Louza, au sud-ouest par la forêt de Kountaida et au sud-est par la forêt de Bouyatas (Fig. 1). Son climat est semi-aride à hiver frais, avec une pluviométrie annuelle de $494 \mathrm{~mm}$, une température annuelle moyenne maximale de $31.47^{\circ} \mathrm{C}$, minimale de $0.91^{\circ} \mathrm{C}$ (O.N.M, 2016). Le sol est de type argilo-brun-calcaire constituant un

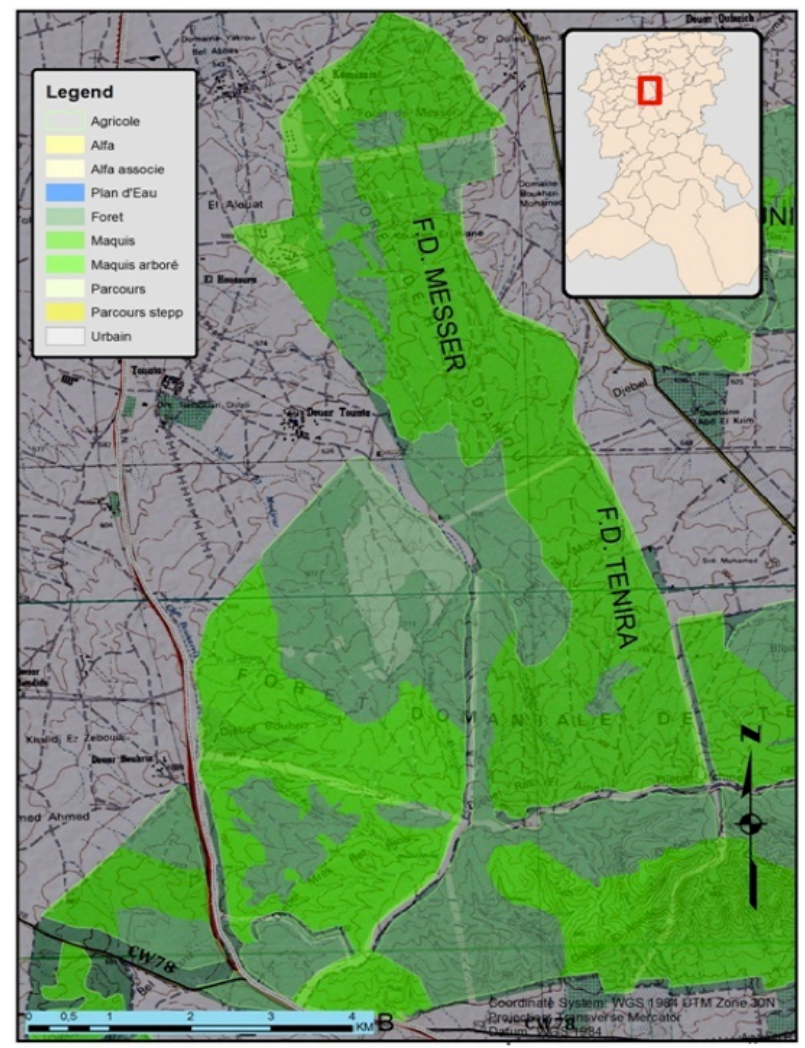

Figure 1. Position géographique de la forêt de Bouhriz (C.F.S.B.A, 2016).

support favorable pour une végétation dominée par le Pin d'Alep (C.F.S.B.A, 2016).

\section{Choix des stations d'échantillonnage}

Sur terrain, quatre stations d'échantillonnage ont été choisies, une station en exposition nordest, un autre sud-est et deux autres stations en exposition sud (tab.1).Le choix de ces stations repose sur la présence des espèces de géophytes dont Tulipa sylvestris L. (fig. 2) fait partie.

\section{Etude floristique}

Dans chaque station, un relevé floristique est réalisé tout en respectant la notion d'aire minimale (Godron et al., 1983), fixé dans notre cas à 100 $\mathrm{m}^{2}$ pendant la période de Mars 2016 jusqu'au Mai 2017.

Pour les espèces non identifiées sur terrain, des échantillons ont été prélevés puis déterminés dans le laboratoire à partir de la flore de Quézel \& Santa $(1962,1963)$.

Les espèces inventoriées sont réparties selon leurs familles (Quézel \& Santa 1962), types biologiques (Raunkiaer, 1934 ; Ellenberg \& Mueller, 1968), types morphologiques et types phytogéographiques (Quézel \& Santa, 1962 ; Ozenda, 1985 ; Bonnier, 1990). 


\begin{tabular}{|l|c|c|c|}
\hline & Coordonnées & Altitude $(\mathrm{m})$ & Exposition \\
\hline S1 & $\mathrm{N}: 35^{\circ} 02,706^{\circ} \mathrm{W}: 000^{\circ} 37,468^{\circ}$ & 673 & Sud - est \\
\hline S2 & $\mathrm{N}: 35^{\circ} 02,480^{\circ} \mathrm{W}: 000^{\circ} 30,799^{\circ}$ & 701 & Nord -est \\
\hline S3 & $\mathrm{N}: 35^{\circ} 02,476^{\circ} \mathrm{W}: 000^{\circ} 37,865^{\circ}$ & 725 & Sud \\
\hline S4 & $\mathrm{N}: 35^{\circ} 02,479^{\circ} \mathrm{W}: 000^{\circ} 37,689^{\circ}$ & 728 & Sud \\
\hline
\end{tabular}

Tableau 1. Coordonnées géographiques, altitudes et expositions des stations d'échantillonnage

Les espèces recensées sont réparties en cinq classes (Durietz, 1920) par rapport à leur fréquence d'apparition $(F)$ dans les stations choisies :

1-classe I espèces très rares : $0<\mathrm{F}<20 \%$

2-classe II espèces rares : $20<\mathrm{F}<40 \%$

3-classe III espèces fréquentes : $40<\mathrm{F}<60 \%$

4-classe IV espèces abondantes : $60<\mathrm{F}<80 \%$

5-classe $V$ espèces constantes : $80<\mathrm{F}<100 \%$

Pour plus de précision et de fiabilité, plusieurs indices ont été calculé, notamment :

L'indice de Sørensen S qui est une mesure très simple de la biodiversité bêta, variant de 0 quand il n'y a pas d'espèces communes entre les deux communautés, à la valeur 1 lorsque les mêmes espèces existent dans les deux communautés.

L'indice de Jaccard $\mathrm{J}$ qui correspond à un test de similarité entre deux stations. Si l'indice $\mathrm{J}$ augmente, un nombre important d'espèces se rencontre dans les deux stations évoquant ainsi que la biodiversité inter station est faible (conditions environnementales similaires entre les habitats). Dans le cas contraire, si l'indice diminue, on ne rencontrera qu'un faible nombre d'espèces présentes sur les deux stations. Ainsi, les espèces pour les deux stations comparés sont totalement différentes indiquant que les différentes conditions de l'habitat déterminent un " turn-over " des espèces importantes (Debello et al., 2007).

L'indice de perturbation (IP) qui permet de quantifier la thérophytisation d'un milieu (Loisel \& Gamila, 1993) pour promouvoir l'état de dégradation des groupements individualisés, un indice de perturbation (IP) a été calculé pour chaque station. Cet indice défini par (Hebrard et al., 1995). est donné par le rapport suivant :

$$
=(\mathrm{Nbr} \text { de Chaméphytes }+\mathrm{Nbr} \text { de }
$$

Therophytes)/(Nbr total des Espéces)

\section{Etude pédologique}

Sur terrain, un prélèvement du sol au niveau de chaque station a été effectué pour une description du substrat à partir des analyses physico-chimiques du sol.

Les paramètres physico-chimiques déterminés sont :

-La texture: La texture d'un sol est révélée par l'analyse granulométrique. Son principe est basé sur la vitesse de sédimentation des particules séparées et dispersées par destruction de leur ciment (calcaire et matière organique).

-Les limons grossiers sont extrapolés par calcul à partir des résultats obtenus (Baize, 1988). Ces résultats sont reportés en fonction des pourcentages des argiles, des limons et des sables dans le triangle des textures (Duchaufour, 1997), pour déterminer cette dernière.

-Le Dosage du calcaire total $\mathrm{CaCO}_{3}$ : ce dosage est fondé sur la réaction caractérisée du carbonate de calcium $\left(\mathrm{CaCO}_{3}\right)$ avec l'acide chlorhydrique $(\mathrm{HCl})$, qui est réalisé à l'aide du calcimètre de Bernard d'après (Baize, 1988).

-Le dosage du calcaire actif : il est réalisé à l'aide d'un réactif spécifique (oxalate d'ammonium), qui attaque une fraction du calcaire total seulement.

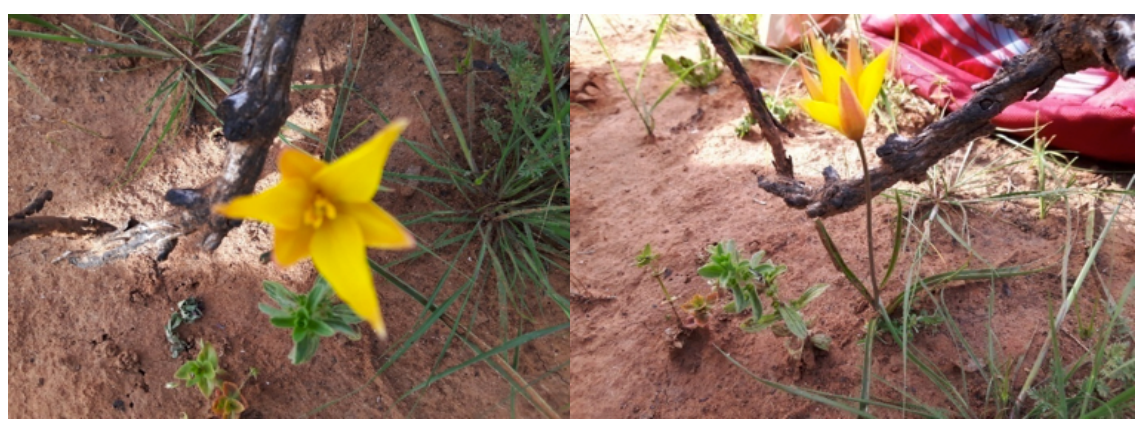

Figure 2. Tulipa sylvestris L. dans la forêt de Bouhriz (Cliché Chihab, 2017) 


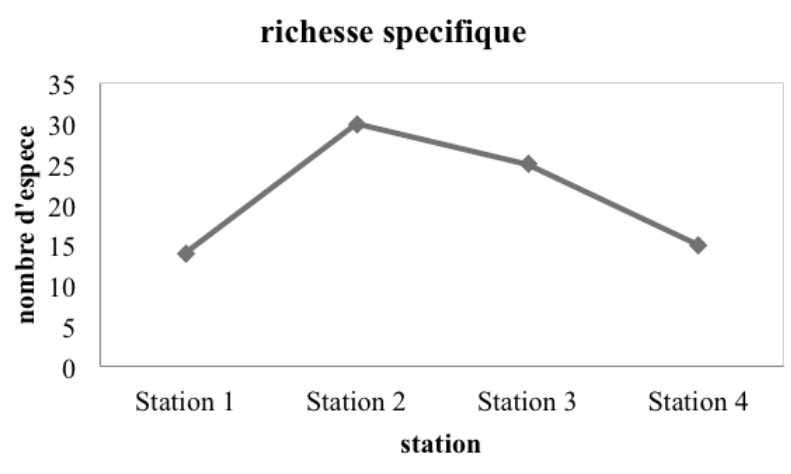

Figure 3. Richesse spécifique des différentes stations

Le calcium extrait est ensuite dosé.

-Le carbone de la matière organique : il est oxydé par bichromate de potassium en présence d'acide sulfurique. En connaissant la quantité de bichromate nécessaire pour cette oxydation, on peut calculer le pourcentage de carbone organique et d'humus dans le sol, (Baize, 1988).

-Pour le $\mathrm{pH}$, le principe consiste à mesurer la force électromotrice d'une solution aqueuse du sol (rapport eau/sol) à l'aide d'un pH-mètre. La conductivité électrique est mesurée à l'aide d'un conductimètre en fonction de la concentration en électrolytes dans une solution d'extraction aqueuse au 1/5 (Richard, 1954). Les compositions cationiques et anioniques de l'extrait des sols ont été réalisées selon la méthode décrite par (Jackson, 1962).

-Pour l'humidité on soumet un échantillon de terre fine, saturée avec de l'eau. On mesure par pesée avant et après séchage à $105^{\circ} \mathrm{C}$.

En effet, la composition de l'horizon de surface, surtout en région méditerranéenne, joue un rôle important sur le comportement de la végétation. $\mathrm{Vu}$ les perturbations humaines, agricoles ou pastorales, la plupart des descripteurs de sol concernent surtout l'horizon superficiel. Ainsi, toutes les caractéristiques morphologiques et analytiques de cet horizon ont une répercussion directe sur la composition floristique (Faraoun et al., 2016).

\section{Etude statistique}

Selon Djebaïli (1984), l'analyse factorielle des correspondances (AFC) permet de traiter conjointement les variables floristiques et les variables écologiques. Ce type d'analyse permet de discriminer de manière objective des entités particulières ou de comparer les stations deux à deux (Bonin \& Tatoni, 1990). La classification hiérarchique ascendante ( $\mathrm{CHA})$ permet de mieux individualiser les limites entre les différents groupements (Benzecri, 1984).

\section{Résultats}

\section{Analyse floristique}

L'inventaire floristique nous a permis de recenser 50 espèces dans l'ensemble des stations choisies dans la forêt de Bouhriz (tab. 2). Le nombre varie de 14 espèces dans la station 1 à 30 espèces dans la station 2 demeurant ainsi la station la plus riche. Cette différence dans le nombre peut être qualifiée comme étant très significative (fig.3).

Les différentes espèces recensées ont été affiliées à leurs familles (tab. 2), on a pu déterminer 21 familles. Les Lamiaceae sont les plus abondants avec un taux de $20 \%$ représentés par 10 espèces, suivies par les Asteraceae avec $14 \%$ et 7 espèces. La troisième place est occupée par les Poaceae et les Fabaceae représentées chacune par 4 espèces et un taux de $8 \%$, suivi par les Liliaceae et les Apiaceae avec $6 \%$ et 3 espèces chacune. Et enfin viennent les autres familles représentées par des taux de $4 \%$ et $2 \%$.

Pour les types biologiques, ce sont les hémicryptophytes qui dominent dans nos stations avec $32 \%$ représentés par 16 espèces (tab. 2), puis les thérophytes ( $20 \%$ et 10 espèces), ensuite les phanérophytes (18\% et 9espèces), les géophytes (16\% et 8 espèces), et en dernier lieu apparaissent les chaméphytes avec 14\% représentés par 7 espèces.

La dominance des hémicryptophytes et des thérophytes remarquée au niveau des stations étudiées, reflète la régression que subissent les formations végétales de la forêt de Bouhriz auparavant dominés par les phanérophytes (Benaouda et al., 2005).

D'un point de vue physionomique, les espèces les plus dominantes sont les espèces vivaces représentées par 35 espèces soit un taux de $70 \%$, suivie par les espèces annuelles avec 9 espèces soit un pourcentage de $18 \%$. Et ensuite les bisannuelles avec 2 espèces seulement soit un pourcentage de $4 \%$.

Concernant les types biogéographiques (fig. 4), pour l'ensemble des stations, on remarque une dominance des espèces méditerranéennes avec un taux de $46 \%$ représenté par 23 espèces. Suivie

Tableau 2. Indices d'abondance-dominance, type biologique, famille et fréquence $(F)$ des différentes espèces recensées dans les quatre stations réalisées dans la forêt de Bouhriz (Algérie nord-occidentale). 


\begin{tabular}{|c|c|c|c|c|c|c|c|}
\hline Espèces & S1 & S2 & S3 & S4 & F \% & Type biologique & Famille \\
\hline Pinus halepensis Mill. & 4-3 & $3-2$ & & $2-2$ & $75 \%$ & Phanérophyte & Pinaceae \\
\hline Quercus ilex L. & $3-2$ & $1-1$ & & & $50 \%$ & Phanérophyte & Fagaceae \\
\hline Olea europaea L. var oléaster Dc & $1-1$ & $1-1$ & & $1-1$ & $75 \%$ & Phanérophyte & Oleaceae \\
\hline Quercus coccifera L. & $1-1$ & $1-1$ & $1-1$ & & $75 \%$ & Phanérophyte & Fagaceae \\
\hline Pistacia lentiscus L. & $3-2$ & $3-2$ & +-+ & $1-1$ & $87,50 \%$ & Phanérophyte & Anacardiaceae \\
\hline Pistacia terebinthus L. & $2-2$ & $2-2$ & & & $50 \%$ & Phanérophyte & Anacardiaceae \\
\hline Urginea maritima L. & $1-1$ & $1-1$ & & & $50 \%$ & Géophyte & Liliaceae \\
\hline Ampelodesma mauritanicum (Bir.) Dur. Et Schiz & $1-1$ & $2-3$ & +-+ & & $62,50 \%$ & Hémicryptophyte & Poaceae \\
\hline Bromus rubens L. & $2-1$ & $2-2$ & & $2-2$ & $75 \%$ & Thérophyte & Poaceae \\
\hline Daucus carota (L.) Thell. & $1-1$ & $1-1$ & & & $50 \%$ & Hémicryptophyte & Apiaceae \\
\hline Plantago lagopus L. & $1-1$ & $1-1$ & $3-1$ & $3-3$ & $100 \%$ & Thérophyte & Plantaginaceae \\
\hline Calycotome spinosa (L.)Link. & $1-1$ & & & & $25 \%$ & Chamaephyte & Fabaceae \\
\hline Stipa tenacissima L. & & $3-3$ & +-+ & $2-2$ & $62,50 \%$ & Géophyte & Poaceae \\
\hline Chamaerops humilis L. & & $2-1$ & & +-+ & $37,50 \%$ & Phanérophyte & Arecaceae \\
\hline Thymus vulgaris $\mathrm{L}$ & & $2-2$ & & & $25,00 \%$ & Chamaephyte & Lamiaceae \\
\hline Thymus officinalis $\mathrm{L}$ & & $2-2$ & & & $25,00 \%$ & Chamaephyte & Lamiaceae \\
\hline Calamintha nepeta (L.)Savi & & $1-1$ & & & $25,00 \%$ & Géophyte & Lamiaceae \\
\hline Ajuga iva (L.) & & $1-1$ & & & $25,00 \%$ & Hémicryptophyte & Lamiaceae \\
\hline Malva sylvestris $\mathrm{L}$. & & $1-1$ & & & $25,00 \%$ & Hémicryptophyte & Malvaceae \\
\hline Rosmarinus officinalis $\mathrm{L}$. & $2-1$ & $2-2$ & $1-1$ & +-+ & $87,50 \%$ & Chamaephyte & Lamiaceae \\
\hline Cistus salviifolius L. & $1-1$ & $2-1$ & & & $50 \%$ & Chamaephyte & Cistaceae \\
\hline Juncus effusus L. & & $2-3$ & & & $25 \%$ & Hémicryptophyte & Juncaceae \\
\hline Foeniculum vulgare (Mill.)Gaertner & & $1-1$ & +-+ & +-+ & $50 \%$ & Hémicryptophyte & Apiaceae \\
\hline Eryngium maritimum L & & & $1-1$ & & $25 \%$ & Hémicryptophyte & Apiaceae \\
\hline Asparagus acutifolius L. & & +-+ & +-+ & & $25 \%$ & Géophyte & Liliaceae \\
\hline Anagallis arvensis L & & & $3-2$ & & $25 \%$ & Thérophyte & Primulaceae \\
\hline Asteriscus maritimus (L.)Less & & & +-+ & $1-2$ & $37,50 \%$ & Hémicryptophyte & Asteraceae \\
\hline Sinapis alba L. & & & $1-1$ & & $25 \%$ & Thérophyte & Brassicaceae \\
\hline Plantago afra $\mathrm{L}$. & & $1-1$ & & & $25 \%$ & Thérophyte & Plantaginaceae \\
\hline Lavandula angustifolia Mill. & & $1-1$ & & & $25 \%$ & Chamaephyte & Lamiaceae \\
\hline Artemisia absinthium $\mathrm{L}$ & & $1-1$ & & & $25 \%$ & Hémicryptophyte & Asteraceae \\
\hline Tulipa sylvestris L & & & +-+ & & $12,50 \%$ & Géophyte & Liliaceae \\
\hline Scilla peruviana L. & & & +-+ & & $12,50 \%$ & Géophyte & Asparagaceae \\
\hline Tripodion tetraphyllum(L)Fourr & & & $2-2$ & +-+ & $37,50 \%$ & Thérophyte & Fabaceae \\
\hline Medicago arabica (L.)Huds. & & & $2-2$ & & $25 \%$ & Thérophyte & Fabaceae \\
\hline Morea sisyrinchiumL & & & $1-1$ & & $25 \%$ & Géophytes & Iridaceae \\
\hline Calendula arvensis $\mathrm{L}$. & & & +-+ & & $12,50 \%$ & Thérophyte & Asteraceae \\
\hline Erica arborea L. & & & $1-1$ & & $25 \%$ & Phanérophyte & Ericaceae \\
\hline Salvia officinalis L. & & & $1-1$ & & $25 \%$ & Chamaephyte & Lamiaceae \\
\hline Scolymus hispanicus L. & & & $1-1$ & & $25 \%$ & Hémicryptophyte & Asteraceae \\
\hline Asteriscus aquaticus (L.)Less. & & $1-+$ & $2-1$ & & $50 \%$ & Hémicryptophyte & Asteraceae \\
\hline Silybum marianum (L.)Gaertn & & & +-+ & +-+ & $25 \%$ & Hémicryptophyte & Asteraceae \\
\hline Glebionis coronoria (L.) Tzvelev & & & +-+ & & $12,50 \%$ & Thérophyte & Asteraceae \\
\hline Reseda alba L. & & & & $1-1$ & $25 \%$ & Hémicryptophyte & résédaceae \\
\hline Mentha rotundifolia L. & & & $2-1$ & & $25 \%$ & Hémicryptophyte & Lamiaceae \\
\hline Ballota hirsuta Benth & & & & $1-1$ & $25 \%$ & Chamaephyte & Lamiaceae \\
\hline Teucrium polium $\mathrm{L}$. & & +-+ & & & $12,50 \%$ & Chamaephyte & Lamiaceae \\
\hline Astragalus Iusitanicus Lamk & & +-+ & & & $12,50 \%$ & Hémicryptophyte & Fabaceae \\
\hline Avena sterilis L. & & $2-2$ & & & $25 \%$ & Thérophyte & Poaceae \\
\hline Scilla bifolia L. & & & & +-+ & $12,50 \%$ & Géophyte & Asparagaceae \\
\hline Total & 14 & 30 & 25 & 15 & & & \\
\hline
\end{tabular}


par 5 espèces eurasiatiques qui représentent $10 \%$ dans l'ensemble des stations, 4 espèces ouest méditerranéennes avec $8 \%$, les espèces cosmopolites avec 3 espèces et un pourcentage de $6 \%$. Les autres types biogéographiques sont représenté par 2 à 1 espèces soit un pourcentage de 4 à $2 \%$.

En effet, malgré la prédominance des espèces méditerranéennes, l'exotisme des autres espèces est bien apparent. L'existence de divers ensembles biogénétiques et biogéographiques majeurs constitue un des facteurs essentiels pour expliquer la richesse des essences en région méditerranéenne.

Pour nos stations inventoriées et d'après les résultats de la fréquence on a pu faire le classement suivant:

Classe I qui indique les espèces très rares représentée par 7 espèces

Classe II qui indique les espèces rares avec 27 espèces

Classe III indiquant les espèces fréquentes avec 7 espèces

Classe IV qui indique les espèces abondantes représentés par 6 espèces

Classe $\mathrm{V}$ qui indique les espèces constantes avec 3 espèces.

En effet, pour les deux premières classes, selon Gégout (1995) la rareté d'une espèce peut être due à trois raisons ; elle est caractéristique d'un autre milieu que celui étudié et y est donc par accident ; elle est "naturellement" rare, sa fréquence est très faible dans toutes les conditions écologiques ; elle a une écologie très étroite - espèce sténooecique : elle est fréquente dans un très faible éventail de milieux et absente ailleurs. Cela peut justifier la présence de Tulipa sylvestris $\mathrm{L}$. dans la catégorie des espèces très rares avec une présence dans une seule station (stations 3 ) même après avoir revue et prospecter tout le périmètre d'étude.

L'indice de similarité de Sorensen et celui de Jaccard (tab. 3) calculés dans notre étude indiquent une présence de relation de similitudes floristiques.

1- la relation entre $S 1$ et $S 2(\beta=0,59$ et $J=0,41)$, S1 et S4 $(\beta=0,41$ et $J=0,26)$, S2 et S4 $(\beta=0,40$ et $J=0,25)$, $S 3$ et $S 4$ présente une forte similitude floristique.

2- la relation entre $S 2$ et $S 3(\beta=0,32$ et $\mathrm{J}=$ $0,19)$, S1 et $S 3(\beta=0,25$ et $J=0,15)$, présente une faible similitude

D'après les résultats du calcul de l'indice de perturbation IP représenté sur le tableau (tab. 4), nous remarquons qu'il est inférieur à $50 \%$ pour les quatre stations de la forêt de Bouhriz, expliquant ainsi une dégradation moyenne des formations végétales engendrée par l'action anthropique à savoir et surtout le surpâturage (ovins, bovins, caprins), les incendies, défrichement, etc.

Avec un taux de $36 \%$, la station 3 explique la forte dégradation que subit la zone d'étude due essentiellement à l'incendie et le surpâturage (fig. 5).

En effet, les perturbations d'origine anthropiques sont responsables pour une large part de l'état actuel des structures de végétation au Maghreb (Quézel \& Barbéro, 1990). Les perturbations causées par l'homme et ses troupeaux sont nombreuses et correspondent à deux situations de plus en plus sévères allant de la matorralisation jusqu'à la désertification passant par la steppisation (Barbero et al., 1990).

\section{Analyse du sol}

Les valeurs moyennes relatives aux différents paramètres physico-chimiques caractéristiques de I'horizon superficiel du sol correspondant aux quatre stations retenues sont représentées sur le Tableau (tab 5.).

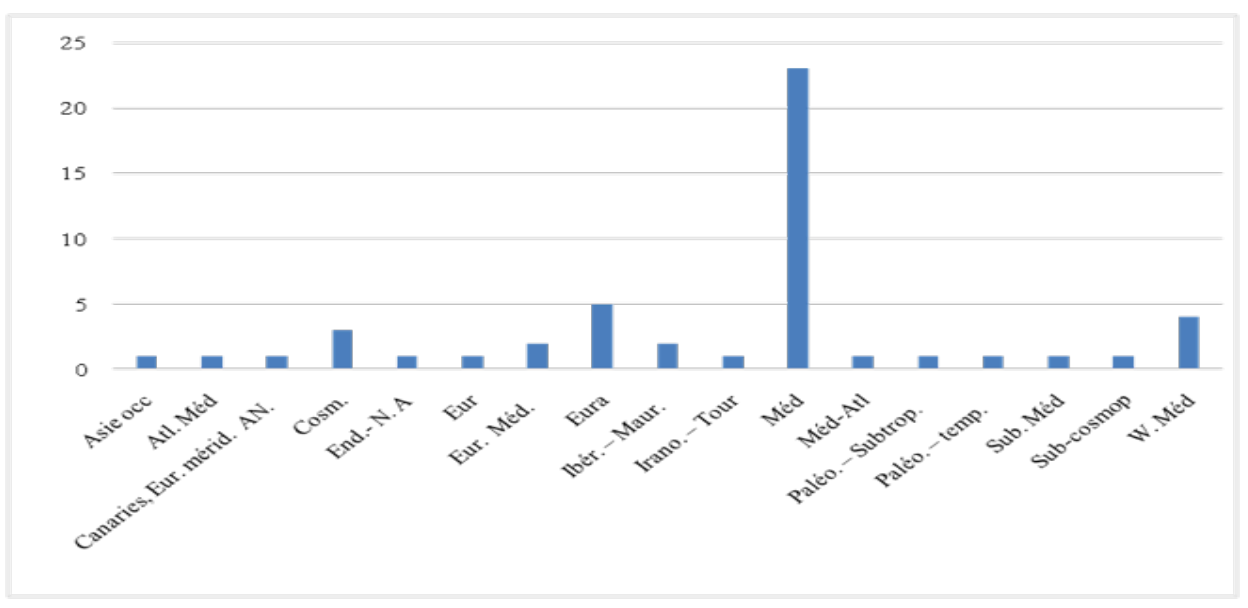

Figure 4. Types biogéographiques des différentes espèces recensées. 


\begin{tabular}{|l|c|c|c|c|c|c|c|c|}
\hline \multicolumn{1}{|c|}{ Station } & \multicolumn{2}{c|}{ S1 } & \multicolumn{2}{c|}{ S2 } & \multicolumn{2}{c|}{ S3 } & \multicolumn{2}{c|}{ S4 } \\
\hline & $\beta$ & $J$ & $\beta$ & $J$ & $\beta$ & $J$ & $\beta$ & J \\
\hline S1 & - & - & & & & & & \\
\hline S2 & 0,59 & 0,41 & - & - & & & & \\
\hline S3 & 0,25 & 0,15 & 0,32 & 0,19 & - & - & & \\
\hline S4 & 0,41 & 0,26 & 0,40 & 0,25 & 0,40 & 0,25 & - & - \\
\hline
\end{tabular}

Tableau 3. Indice de similarité de Sorensen " $\beta$ »et de Jaccard «J》

\begin{tabular}{ccccc}
\hline Station & S1 & S2 & S3 & S4 \\
\hline IP & $35,71 \%$ & $33,33 \%$ & $36 \%$ & $26,66 \%$ \\
\hline
\end{tabular}

Tableau 4. Indice de perturbation IP des stations étudiées

Nous notons une variabilité dans la profondeur du sol et cela met en évidence les caractères différentiels des stations étudiées, la profondeur augmente de $10 \mathrm{~cm}$ dans la station 1 à $18 \mathrm{~cm}$ dans la station 4 déterminant ainsi des sols superficiels.

Les pentes sont variables, on remarque des stations à faible pente (station 2 et 4 ) à moyenne (station 1).

Le taux d'humidité varie entre $4,2 \%$ à la station 4 avec son exposition sud et $9,42 \%$ à la station 2 au versant nord-est.

L'élément le plus remarquable c'est le pourcentage élevé du sable pour l'ensemble des quatre stations avec des valeurs de $65,3 \%$, $70,29 \%, 60,23 \%$ et $51,55 \%$, respectivement pour les stations 1, 2, 3 et 4 .

Les limons et les argiles sont faiblement présents. Par apport au sable, maison note un taux de limons de $32,28 \%$ pour la station 4 et un taux d'argile de $16,42 \%$ pour la station 2

Concernant la texture du sol, trois stations sont sablo-limoneuses (station 1, 2 et 3) une station des limono-sableuse (station 4), La couleur des échantillons du sol et variable et diversifier allons du marron (station 1, 2 et 3 ) au marron claire (station 4)

Le $\mathrm{pH}$ est légèrement alcalin pour les stations $1,2,3$ et fortement alcalines avec une valeur maximum de 8,18 pour la station 4 .

Pour la conductivité électrique, les résultats obtenus indiquent des sols non salés (C. $E^{<} 0,6 \mathrm{~ms} /$ $\mathrm{cm})$.

Le taux du calcaire total représente des valeurs respectives de $0,45 \%, 6,36 \%, 0,15 \%, 8,18 \%$ pour les stations $1,2,3$ et 4 déterminant ainsi des sols modérément calcaires avec un maximum enregistre dans la station 4.

La teneur en calcaire actif affiche des valeurs minimales de $0,12 \%$ pour la station 1 et un maximum de 2,62 \% pour la station 4 .

Les résultats de la teneur en matière organique fait apparaitre une richesse dans la station 4 avec une valeur de l'ordre de $6,21 \%$. Pour les autres stations, elles sont moyennement riches en matière organique avec un pourcentage qui varie entre $2,77 \%$ à $3,44 \%$.

$\mathrm{pH}$ : potentiel hydrique, C.E : conductivité électrique, M.O : matière organique, SL :sablolimoneuses, LS : limono-sableuses, C.T : calcaire total, C.A : calcaire actif

\section{Traitement statistique}

L'analyse factorielle des correspondances (AFC) effectuée pour nos quatre stations permet de montrer la relation entre les différents types biologiques et les paramètres édaphiques (fig.6).

Le plan factoriel Fact. 1 avec une valeur d'inertie de $63,36 \%$ plus importante que l'axe Fact.2 avec une valeur de $32,93 \%$, donc l'interprétation se fera en fonction de l'axe 1 qui présente des informations plus significatives.

Le choix des deux groupes a été réalisé selon la particularité et la contribution aux deux axes factoriels de chaque station avec ses paramètres (floristiques et édaphiques), ce choix s'est reposé sur la classification hiérarchique ascendante $(\mathrm{CHA})$ réalisée en parallèle. Deux groupes se sont distingués par rapport à l'axe $1,(A)$ du côté positif et (B) du côté négatif.

Le groupe $(A)$ définie par les stations 1et 4 où les phanérophytes avec leurs strates arborées et arbustives tel que les espèces Pinus halepensis $L$. et Quercus ilex L. dominent le groupement avec des taux de recouvrement (RCT) plus importants variant de 80 à $85 \%$, lié à l'humidité $(\mathrm{Hm})$, le sable $(\mathrm{Sb})$, l'argile (Ar), et le calcaire actif (CA).

La dominance de ces strates joue un rôle important dans la protection de la flore surtout 


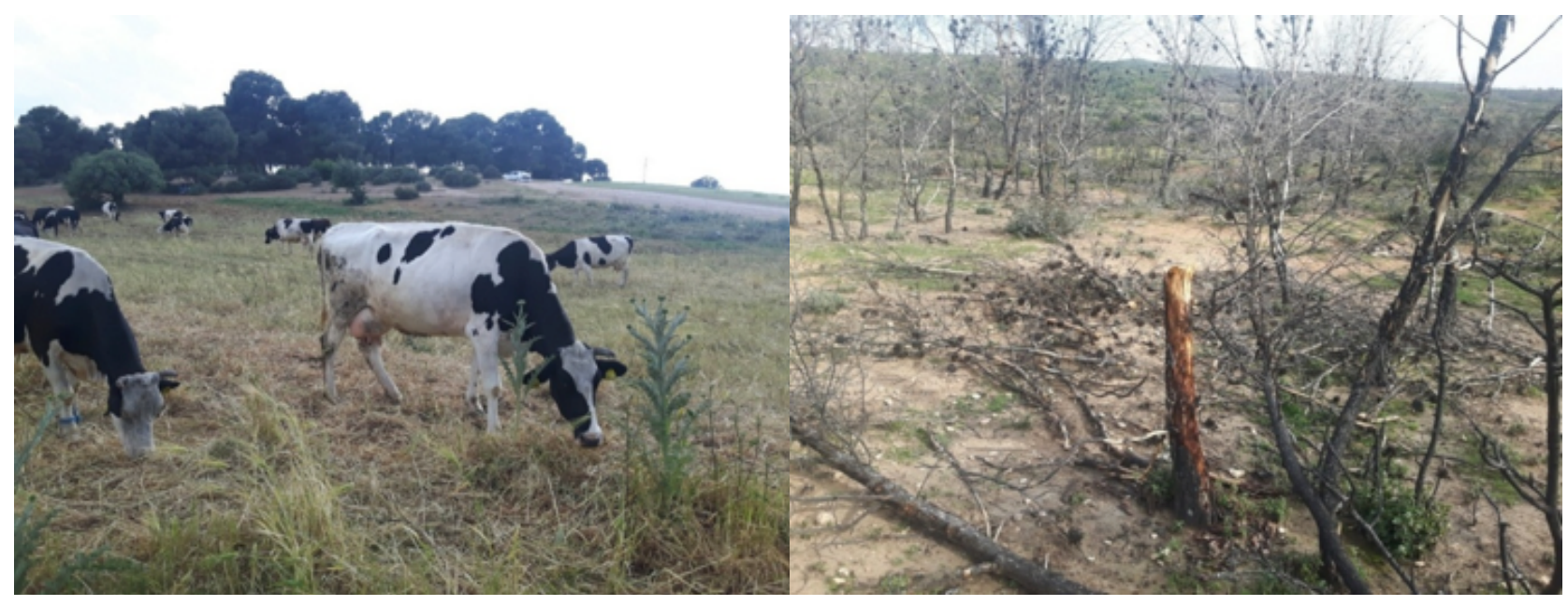

Figure 5. Pâturage excessif des bovins et dégradation par incendie dans la zone d'étude (Cliché Chihab, 2017)

\begin{tabular}{|l|c|c|c|c|}
\hline & Station 1 & Station 2 & Station 3 & Station 4 \\
\hline Profondeur (cm) & 10 & 16 & 15 & 18 \\
\hline Couleur & Marron & Marron & Marron & Marron claire \\
\hline Texture & SL & SL & SL & LS \\
\hline Pente (\%) & 10 & 2 & 6,7 & 4,2 \\
\hline Humidité (\%) & 8,25 & 9,42 & 26,57 & 32,28 \\
\hline Limons (\%) & 25,52 & 13,28 & 13,19 & 16,16 \\
\hline Argiles (\%) & 9,16 & 16,42 & 60,23 & 51,55 \\
\hline Sables (\%) & 65,3 & 70,29 & 7,8 & 8,18 \\
\hline pH & 7,7 & 7,74 & 0,03 & 0,06 \\
\hline C.E (ms/cm) & 0,06 & 0,07 & 3,44 & 6,21 \\
\hline M.O (\%) & 3,44 & 2,27 & 0,15 & 8,18 \\
\hline C.T (\%) & 0,45 & 6,36 & 1,25 & 2,62 \\
\hline C.A $(\%)$ & 0,12 & 1,37 & 5 \\
\hline
\end{tabular}

Tableau 5. Résultats des analyses pédologiques des échantillons de sol pour les stations d'étude réalisées dans la forêt de Bouhriz.

contre le pâturage excessive (surpâturage).

Du coté négative, deux sous groupements se distinguent $\left(B_{1}\right)$ et $\left(B_{2}\right)$ à l'intérieur du groupe $(B)$.

Le premier $\left(B_{1}\right)$, qui renferme la station 3 (station abritant Tulipa sylvestris L.) où dominent les thérophytes et les géophytes, est rattaché au taux de la conductivité électrique(CE) et au calcaire total (CT). En effet, cette station témoigne de la dégradation que subissent les formations végétales dans la forêt de Bouhriz où l'installation d'espèce de thérophytisation tel que Plantago lagopus L., Anagallis arvensis L., Tripodion tetraphyllum L., Medicago arabica L. est observée. Cela explique l'augmentation de l'indice de perturbation (de l'ordre de $36 \%$ ) dans cette station dû essentiellement à des actions anthropozoogènes (fig. 5).

Le deuxième sous-groupe $\left(B_{2}\right)$ renferme la station 2 où dominent les hémicryptophytes et les chamaephytes représentés essentiellement par Thymus officinalis L., Rosmarinus officinalis L. et Cistus salviifolius $L$. ainsi que les géophytes tel que ; Stipa tenacissima L. en plus cette station regroupe une diversité dans le nombre de famille (FAM) rattachée au taux des limons (Li), le potentiel hydrique $(\mathrm{pH})$, et la matière organique $(\mathrm{MO})$.

D'une manière générale, du côté positif vers le côté négatif sur l'analyse factorielle des correspondances on assiste à une dégradation.

\section{Discussion}

Tulipa sylvestris L. était localisé dans un terrain très dégradé touché par une action anthropozoogène flagrante (fig. 5). Cette situation 


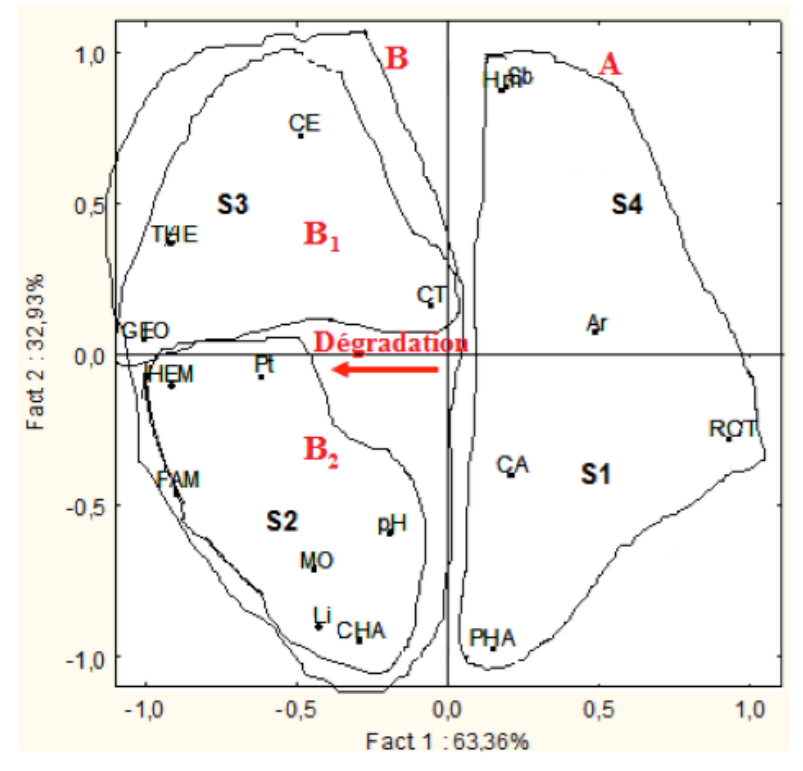

Figure 6. Analyse factorielle des correspondances (AFC). GEO : géophytes ; HEM : hémicryptophytes ; THE : thérophytes ; pt : pente ; CT : calcaire total ; CA : calcaire actif ; Ar : argile ; RCT : recouvrement ; FAM : Familles ; $\mathrm{MO}$ : matière organique ; $\mathrm{Li}$ : limon ; $\mathrm{pH}$ : potentiel hydrique ;CE : conductivité électrique ; $\mathrm{Sb}$ : sable ; Hm : humidité $\mathrm{CHA}$ : chaméphytes ; PHA :phanérophytes ; S : station.

alarmante de l'habitat menace directement l'existante et la pérennité de l'espèce, en effet, la place de l'homme dans l'environnement méditerranéen est aujourd'hui importante puisque ses impacts et ses choix de gestion déterminent les dynamiques des écosystèmes et la magnitude des phénomènes de raréfaction ou d'extinction des espèces (Médail \& Diadema, 2006).

Les analyses du sol ont montré que le substrat est caractérisé par un pH légèrement alcalin, des sols non salés, en ce qui concerne la texture, nous avons remarqué que la texture sablo-limoneuse et la texture limoneux-sableuse prédominent. Ces deux textures sont typiques des sols du site d'étude (Chihab et al., 2017), des sols modérément calcaires moins riches en matière organique.

Quoique la pression anthropique soit certainement un facteur majeur de son déclin (Lotter, 1999), la collecte du bois de feu, considéré comme l'unique source d'énergie en milieu rural, contribue de manière considérable à la destruction de la couverture végétale (Cherifi et al., 2011), toutefois, les formations d'équilibre primaire le climax des biogéographes - sont souvent impuissantes à se régénérer spontanément en cas de dégradation massive par l'homme (Toubert, 1997).

Dans cet écosystème fragilisé, l'action anthropique reste le principal facteur de dégradation (Cherifi et al., 2011). Ainsi, le surpâturage peut causer une dégradation de la qualité de l'eau, modifie la structure des sols et altère la structure et la composition des communautés végétales (Hamel et al., 2006). Certainement, l'activité anthropogène de par le surpâturage, le défrichement et l'éradication des ligneux contribue à l'atteinte négative de la biodiversité existante (Mimoune, 1995; Hadjab, 1998; Daget \& Poissonet, 1997). De ce fait, la protection des espèces rares tel que la Tulipe exige une stratégie de conservation intégrée (Falk, 1992; Frankel et al., 1995).

En effet l'activité d'élevage engendre un surpâturage, une réduction de la couverture végétale et une destruction de la flore originale par broutage, par piétinement ou même par prolifération de certain taxon non palatable et témoignent d'un déséquilibre qui sévit (Zedam, 2015). Floret et al., (1990) signalent que plus un système est influencé par l'homme (surpâturage, culture), plus les thérophytes prennent de l'importance. Car souvent ce type de perturbation est souvent incriminé dans la réduction de la biodiversité (Dolek \& Geyer, 1997).

\section{Conclusions}

L'étude floristique de l'ensemble des stations a permis de recenser 50 espèces associées aux géophytes, dont 25 espèces associées à Tulipa sylvestris L., cette plante menacée et rare localisée dans la partie sud de la forêt de Bouhriz (région de Sidi Bel Abbes), se développe à une altitude de $725 \mathrm{~m}$ sur des substrats à texture limonosableuse, peu humide avec un $\mathrm{pH}$ légèrement alcalin, moyennement riches en matière organique et modérément calcaire.

La station où l'espèce se développe, en se basant sur l'analyse factorielle des correspondances (AFC), présente une forte thérophytisation caractérisée par la dominance des espèces indicatrices de dégradation tel que Plantago lagopus L., Anagallis arvensis L., Tripodion tetraphyllum $\mathrm{L}$. et Medicago arabica $\mathrm{L}$. Ces résultats ont été confirmés par un indice de perturbation de l'ordre de $36 \%$.

D'une manière générale, la disparition et la rareté de Tulipa sylvestris L. est due essentiellement à une action anthropozoogène. Le surpâturage associé aux incendies répétés ainsi que l'ouverture de la forêt au tourisme peuvent sans doute causer une importante dégradation des formations végétales.

En effet, cette étude nous incite à trouver des solutions de protection et de conservation efficace 
et durable pour la pérennité de Tulipa sylvestris $L$. en particulier et les formations végétales dans la région en générale.

\section{Références bibliographiques}

Alcaraz, C. (1980). La végétation de l'Ouest algérien. Thèse de doctorat d'État. Université de Perpignan.

Baize, D. (1988). Guide des analyses courantes en pédologie (éd. INRA). Paris.

Barbero, M., Loisiel, R. \& Quezel, P. (1990). Les apports de la phyto-écologie dans l'interprétation des changements et perturbations induits par l'homme sur les écosystèmes forestiers méditerranéens. Forêts méditerranéennes, 2: 194-215.

Benaouda, Z., Mehdadi, Z. \& Bouchaour, I. (2005). Influence pédoclimatique sur l'évolution des formations forestières en zone semi-aride (cas de la forêt de Tenira, Ouest algérien). Sécheresse, 16 (2), 115-20.

Ben Abdellah, (2010). Analyse phytoécologique des groupements à thuya (Tetraclinis articulata (Vahl) Masters) et à chêne vert (Quercus rotundifolia Lam.) dans les monts de Tlemcen (Algérie occidentale). Ecologia mediterranea, 36 (2), 99-115.

Benzecri, JP. (1984). L'analyse des correspondances. Paris: Dunod editions.

Bonin, G. \& Tatoni, T. (1990). Réflexions sur l'apport de l'analyse factorielle des correspondances dans l'étude des communautés végétales et de leur environnement. Ecologia Mediterranea, 16: 403-14.

Bonnier, G. (1990). La grande flore en couleur. Édition Belin, tomes 1-2-3-4, Paris, France, 1401 p.

Cherifi, K., Mehdadi, Z., Latreche, A. \& Bouiadjara, S.B. (2011). Impact de l'action anthropozoogène sur l'écosystème forestier du mont de Tessala (Algerie occidentale) Article de recherche. Sècheresse, 22: 197-206.

Chihab, M., Bouzidi, M. A., Latreche, A. \& Dif, M. M. (2017). Physicochemical Characterization of Geophytes Soil Habits in Sidi Bel Abbes area (Algeria, NW). J. Appl. Environ. Biol. Sci., 7(5): 77-84.

Daget, P. \& Poissonet, J. (1997). Biodiversité et végétation pastorale. Revue Elev. Méd Vet. Pays Trop, 50(2): 141- 144.

Debello, F. Lepš, J. \& Sebastià, M. T. (2007). Grazing effects on the species-area relationship: Variation along a climatic gradient in NE Spain. Journal of Vegetation Science, 18: 25-34.

Direction generale des forets conservation des forets de la wilaya de sidi bel abbes (2016). Fiche descriptive de la forêt de Bouhriz.

Djebaili, S. (1984). Steppe algerienne, phytosociologie et ecologie. Recherche phytosociologique et écologique sur la végétation des hautes plaines steppiques et de l'Atlas saharien. Alger: Office des publications universitaires (OPU).

Dolek, M. \& Geyer, A. (1997). Influence of management on butterflies of rare grassland ecosystems in Germany. J. Insect Conserv, 1: 125-130.

Duchaufour, PH. (1997). Abrégé de Pédologie. Sol, végétation, environnement. Masson, Paris, $291 \mathrm{p}$.
Duretz, E. (1920). Zun methodologischen grundlage der modern pflaugenziologie. Upsala $252 \mathrm{p}$.

Ellenberg, S. \& Mueller, P. (1968). Akey of Raunkiaerplant use forms with revised. Ber. Geobot. Inst.ETH. Stift. Rubel. Zurich, 37: 56-73.

Falk, D. A. (1992). From conservation biology to conservation practice: strategies for protecting plant diversity. In Conservation Biology. The Theory and Practice of Nature Conservation, Preservation and Management.

Faraoun, F., Bouzidi, M.A., Attaoui, I., Latreche, A., Meliani, H. \& Benyahia M. (2016). Caractérisation des formations végétales à Ampelodesmos mauritanicus (Poir.) D. \& S. sur Djebel Tessala, Algérie occidentale, Afrique Science, 12(5): 326-335.

Floret, C., Galan, M.J., Le Floc'h, E., Orshan, G. \& Romane, F. (1990). Dynamics of holm oak (Quercus ilex L.) coppies after clear cutting in southen France. Vegetation, 11: 99-100.

Frankel, O. H., Brown, A. H. D. \& Burdon, J. J. (1995). The Conservation of Plant Biodiversity. Cambridge University Press, Cambridge.

Gegout J.C. (1995). Etude des relations entre les ressources minérales du sol et la végétation forestière dans les vosges. Thèse Doc. ENGREF. Nancy I. 215p.

Godron, M., Daget, P., Emberger, L., Long, G., Le Floch, E., Poissonet, J., Sauvage, C. \& Wacquant J.P. (1983). Code pour le relevé méthodique de la végétation et du milieu. Ed. C.N.R.S. Paris. 296p.

Greuter, W. (1991). Botanical diversity, endemism, rarity, and extinction in the Mediterranean area: ananalysis based on the published volumes of Med-Checklist. Botanika Chronika, 10: 63-79.

Guinochet, M. (1980). Essai sur quelques syntaxons des Cisto-Rosmarinetea et des Quercetea ilicis d'Algérie et de Tunisie. Phyto-écologia, 7: 227.

Hadjab, M. (1998). Aménagement et protection des milieux naturels dans la cuvette centrale du Hodna (Algerie). Thèse de Doctorat, université d'Aix Marseille 1-université de Provence (France).237 p + annexes.

Hadjadj Aoul, S. (1995). Les peuplements du Thuya de berbérie (Tetraclinis articulata, Vahl, Master) en Algérie: phytoécologie, syntaxonomie et potentialités sylvicoles. Thèse. Doc. D'État, Univ. Aix-Marseille III.

Hamel, C., Reisz R., Fortney, G., Jones, R. \& Pietruszewski, D (2006). Conservation Area Plan for the Tallgrass Aspen Parkland, Conservation de la nature Canada, Région du Manitoba, Winnipeg (Manitoba)/The Nature Conservancy, Karlstad Field Office, Karlstad (Minnesota), 70 p.

Hébrard, J.P., Loisel, R., Roux, C., Gomila, H. \& Bonin, G. (1995).Incidence of clearing on phanerogamic and cryptogamic vegetation in South-Eastern France: disturbance indices, in Bellan D., Bonin G., Emig C. (Eds.), Functioning and dynamics of natural and perturbed ecosystems, Lavoisier, Paris 1995, pp. 747758.

Jackson, M. (1962). Soil chemical analysis (éd. Constable and Comp). England.

Joffre, R., Rambal, S. \&Ratte, J.P. (1999). The dehesa system of southern Spain and Portugal as a natural ecosystem mimic. Agro forestry Syst, 45: 57-79. 
Kunin, W.E. \& Gaston, K.J. (1993). The biology of rarity: patterns, causes and consequences. Trends in Ecology and Evolution, 8: 298-301.

Lacroix, P., Le Bail, J., Hunault, G., Brindejonc, O., Thomassin, G., Guitton, H., Geslin, J., \&Poncet, L. (2008). Liste «rouge» régionale des plantes vasculaires rareset/ou menacées en pays de la Loire, Nantes. Conservatoire Botanique National de BrestAntenne régionale des Pays de la Loire, Région des Pays de la Loire, 48 p., + annexes.

Lacroix, P., Le Bail, J., Brindejonc, O., et al. (2009). Liste «rouge» départementale des plantes vasculaires rares et/ou en régression en Loire-Atlantique. Conservatoire Botanique National de Brest, Conseil Général de Loire-Atlantique, 46 p. + annexes.

Lavergne, S., E. Garnier, \& Debussche, M. (2003). Do rockendemic and widespread plant species differ underthe leaf-height-seed plant ecology strategy scheme? Ecol. Lett., 6: 398-404.

Lavergne, S., W. Thuiller, J. Molina \& Debussche, M. (2005). Environmental and human factors influencingrare plant local occurrence, extinction and persistence: a 115 years study in the Mediterranean region. J. Biogeogr., 32: 799-811.

Levinton, J. S. (1982). Estimatingstasis: can a null hypothesis be too null? Paleobiology, 8: 307

Loisel, R. \& Gamila, H. (1993). Traduction des effets du débrous-saillement sur les écosystèmes forestiers et pré-forestiers par un indice de perturbation. Ann. Soc. Sci. Nat. Archéol. de Toulon du var. 123-132.

Lotter, A. (1999). Late-glacial and Holocene vegetation history and dynamics as shown by pollen and plant macrofossil analyses in annually laminated sediments from Soppensee, central Switzerland, Veget. Hist. Archaeobot., 8: 165-184.

Magnanon, S. (1993). Liste «rouge» des espèces végétales rares et menacées du Massif armoricain. E.R.I.C.A. $\mathrm{n}^{\circ} 4,1-22$.

Magnanon, S. \& Hardy, F. (1999). Stratégie intégrée de conservation des taxons les plus rares et les plus menacés du Massif armoricain. Bulletin de la Société Botanique du Centre-Ouest, Nouvelle série, $\mathrm{n}^{\circ}$ spécial $\mathrm{n}^{\circ} 19,355-378$.

Marc, M. (1916). Les forêts d'Algérie. Ed. Jourdan. Alger. $331 \mathrm{p}$.

Medail, F. \& Diadema, K. (2006). Biodiversité végétale méditerranéenne et anthropisation: approches macroet micro-régionales. Ann. Géogr., 651: 618-640.

Mimoune, S. (1995- Gestion des sols salés et désertification dans une cuvette endoréique d'Algerie (Sud Du Chott El Hodna). Thèse de Doctorat, université d'Aix Marseille $1204 \mathrm{p}$.

Murray, B.R., Thrall, P.H., Gill, A.M. \& Nicotra, A.B. (2002). How plant life-history and ecological traitsrelate to species rarity and commonness at varying spatial scales. Austral Ecology, 27: 291-310.

Myers, N., Mittermeier, R., Mittermeier, C., Da Fonseca, G. \& Kent, J. (2000). Biodiversity hotspots for conservation priorities. Nature, 403: 853-858.
Office National De la Météorologie (O.N.M.) (2017). Recueil des données climatiques de la wilaya de Sidi Bel Abbes. Feuilles de relevés quotidiennes de la période 1980-2016. Alger.

Olivier, L., Galland, J.P., Maurin, H. \& Roux, J.P. (1995). Livre rouge de la flore menacée de France. Tome I: espèces prioritaires. Muséum National d'Histoire Naturelle, Conservatoire Botanique National de Porquerolles, Ministère de l'Environnement, Collection Patrimoines Naturels, vol. 20, 486 p + annexes.

Ozenda, P. (1985). Flore du Sahara septentrional et centre. CNRS, Paris, France, $441 \mathrm{p}$.

Quezel, P. \& Santa, S. (1962). Nouvelle flore de l'Algérie et des régions désertiques méridionales. Tome I. Ed. CNRS. Paris. 197p.

Quezel, P. \& Santa, S. (1962). Nouvelle flore de l'Algérie et des régions désertiques méridionales. Tome I. Ed. CNRS. Paris. 565p.

Quezel, P. \& Santa, S. (1963). Nouvelle flore de l'Algérie et des régions désertiques méridionales. Tome II. Ed. CNRS. Paris. 605p.

Quezel, P. (1964). L'endémisme dans la flore de l'Algérie. C. R. Soc. Biogeo., 137-149.

Quézel, P. (1985). Definition of the Mediterranean region and the origin of its flora. In: Plant conservation in the Mediterranean area (Ed. Gomez-Campo C.), pp. 9-24. Geobotany, Dordrecht, The Netherlands.

Quézel, P. \& Barbero, M. (1990). Les forêts méditerranéennes, problèmes posés par leur signification historique, écologique et leur conservation. Acta Botanica Malacitana, 15: 145-178.

Raunkiaer, C. (1934). The life forms of plants and statistical plant. Edition Claredon Press, Oxford, 632 p. Richard, L.A. (1954). Diagnosis and improvement of saline and alkali soils. Édit. US Department of Agriculture, Agricultural Handbook $n^{\circ} 60$, Washington (USA), $160 \mathrm{p}$.

Sala, O.E., Chapin, F.S.I., Armesto, J.J., Berlow, E., Bloomfield, J., Dirzo, R., Huber-Sanwald, E., Huenneke, L.F., Jackson, R.B., Kinzig, A., Leemans, R., Lodge, D.M., Mooney, H.A., Oesterheld, M., Poff, N.L., Sykes, M.T., Walker, B.H., Walker, M. \& Wall, D.H. (2000). Global biodiversity scenarios for the year 2000. Science, 287: 1770-1774.

Toubert, P. (1997). L'homme et l'environnement dans le monde méditerranéen: le regard du médiéviste. In: Regards sur la Méditerranée. Actes du 7ème colloque de la Villa Kérylos à Beaulieu-sur-Mer les 4 \& 5 octobre 1996. Paris: Académie des Inscriptions et Belles- Lettres, pp. 113-133. (Cahiers de la Villa Kérylos, 7).

Verlaque R., Medail, F. \& Aboucaya, A. (2001). Valeur prédictivedes types biologiques pour la conservation de la flore méditerranéenne. C. R. Acad. Sci. Paris, Sciences de la Vie/Life Sciences, 324: 1157- 1165.

Zedam, A. (2015). Thèse de Doctorat en sciences, biologie végétale, Etude de la flore Endémique de la zone humide de chott el Hodna inventairepréservation. Novembre 2015 chapitre VI page 128. 